\title{
MATRIX INVERSION AND DIGRAPHS: THE ONE FACTOR CASE*
}

\author{
T. BRITZ ${ }^{\dagger}$, D. D. OLESKY ${ }^{\ddagger}$, AND P. VAN DEN DRIESSCHE ${ }^{\dagger}$
}

\begin{abstract}
The novel concept of a cyclic sequence of a digraph that has precisely one factor is defined, and is used to characterize the entries of the inverse of a matrix with such a digraph. This leads to a characterization of a strongly sign-nonsingular matrix in terms of cyclic sequences. Nonsingular nearly reducible matrices are a well-known class of matrices having precisely one nonzero diagonal, and a simple expression for the entries of the inverse of such a matrix in terms of cyclic sequences is derived. A consequence is that a nonsingular nearly reducible matrix is strongly signnonsingular. Several conditions that are equivalent to the inverse of a nonsingular nearly reducible matrix being nearly reducible are obtained.
\end{abstract}

Key words. Inverse matrix, Digraph, Sign pattern, Strongly sign-nonsingular matrix, Nearly reducible matrix, Minimally strongly connected digraph.

AMS subject classifications. 05C50, 15A09, 05C20.

1. Introduction. For several decades, matrix inversion has been described in terms of digraph properties; see for example $[4,6,12,13]$ and references therein. This paper focuses on a special class of matrices, namely $m \times m(m \geq 2)$ matrices with precisely one nonzero diagonal. In terms of digraphs, this is equivalent to the digraph of a matrix having precisely one factor. For such matrices, matrix inversion may be characterized in a relatively simple manner by means of digraph properties, as described in the main result of Section 2, namely Theorem 2.4, by employing the novel concept of a cyclic sequence. In Section 3, Theorem 3.3 provides a necessary and sufficient condition for the matrices associated with a given digraph with precisely one factor to all have inverses with a common digraph. This result is extended in Section 4 by Theorem 4.3, which also characterizes strongly sign-nonsingular matrices. Although similar characterizations may be found elsewhere (see [4, Chapter 7] for an overview), the present characterization offers new necessary and sufficient conditions. Furthermore, this characterization does not depend on the usual normalization condition [4] that the main diagonal of the matrix in question has only negative entries.

An $m \times m$ matrix is nearly reducible if it is irreducible and becomes reducible whenever any nonzero entry is replaced by zero. Such matrices have been shown to have interesting properties, many of which are described by Brualdi and Ryser [3, Section 3.3]. Section 5 is devoted to the study of the digraphs of such matrices (minimally strongly connected digraphs) and the structure of the digraphs of the inverses of these matrices. Hedrick and Sinkhorn [7] proved that nonsingular nearly reducible matrices have precisely one nonzero diagonal. Thus the inversion characterization in

${ }^{*}$ Received by the editors 22 September 2003. Accepted for publication 23 May 2004. Handling Editor: Bryan L. Shader. This work was supported by NSERC Discovery Grants. T. Britz was also supported by a PIMS Postdoctoral Fellowship.

${ }^{\dagger}$ Department of Mathematics and Statistics, University of Victoria, Victoria, BC V8W 3P4, Canada (britz@math.uvic.ca, pvdd@math.uvic.ca).

${ }^{\ddagger}$ Department of Computer Science, University of Victoria, Victoria, BC V8W 3P6, Canada (dolesky@cs.uvic.ca). 
Theorem 2.4 may be applied to these matrices, and Theorem 5.8 presents a particularly simple description of the inverse of a nonsingular nearly reducible matrix in terms of cyclic sequences. From this description, it follows directly that each nonsingular nearly reducible matrix is strongly sign-nonsingular. Furthermore, the inversion of a nonsingular nearly reducible matrix has an interesting digraph analogue. In particular, Theorem 5.15 describes how the digraphs of a nonsingular nearly reducible matrix and its inverse determine each other by digraph operations. Proposition 5.16 shows that this dual correspondence is valid not only for the digraphs of the two matrices, but also for their sign patterns. This is true despite the fact that the inverse of a nonsingular nearly reducible matrix is neither necessarily nearly reducible nor strongly sign-nonsingular. Interestingly, it is seen in Theorem 5.18 that the inverse is nearly reducible if and only if it is strongly sign-nonsingular.

2. Matrices with precisely one nonzero diagonal. We begin with some digraph terminology and introduce the concept of a cyclic sequence, which plays an important role throughout this paper. The family $\mathcal{D}_{1}$ consists of all (finite) digraphs $D$ with precisely one factor $\mathcal{C}_{D}=\left\{C_{1}, \ldots, C_{s}\right\}$; that is, $\mathcal{C}_{D}$ is a set of vertex-disjoint cycles that cover all vertices $V(D)$. It is assumed that $|V(D)| \geq 2$. The $\operatorname{arcs} E(D)$ of a digraph $D \in \mathcal{D}_{1}$ may be partitioned into two non-empty classes, namely the arcs that are contained in one of the cycles of $\mathcal{C}_{D}$, and those that are not. Denote the former by $E\left(\mathcal{C}_{D}\right)=E\left(C_{1}\right) \cup \cdots \cup E\left(C_{s}\right)$. If $v$ is a vertex of $V(D)$, then $v$ is contained in precisely one cycle of $\mathcal{C}_{D}$. This is the cycle $\mathcal{C}_{D}(v)$ given by

$$
v \rightarrow \sigma(v) \rightarrow \sigma(\sigma(v)) \rightarrow \cdots \rightarrow \sigma^{-1}(v) \rightarrow v
$$

where $\sigma$ is the permutation of $V(D)$ determined by the factor $\mathcal{C}_{D}$, i.e., $\sigma(u)=v$ if and only if $(u, v) \in E\left(\mathcal{C}_{D}\right)$.

To avoid any confusion between $\sigma$ and $\sigma^{-1}$ in the following, we use the notation $n:=\sigma$ and $f:=\sigma^{-1}$ (for the "next" and "former" permutations in $D$ ). It follows that $v=n(f(v))=f(n(v))$ for each vertex $v \in V(D)$, and that if $(u, v)$ is contained in $E\left(\mathcal{C}_{D}\right)$, then $(u, v)=(f(v), n(u))$. The permutations $n$ and $f$ are illustrated in Figure 2.1, in which $\mathcal{C}_{D}$ consists of three 3 -cycles.

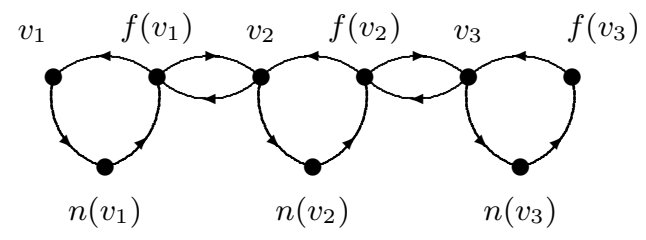

FIG. 2.1. The "next" and "former" permutations

A cyclic sequence in $D \in \mathcal{D}_{1}$ of length $s \geq 1$ from vertex $v_{1}$ to vertex $f\left(v_{s}\right)$ is a sequence of $s$ distinct vertices $\left(v_{1}, \ldots, v_{s}\right)$ of $D$ such that if $s \geq 2$, then $\left(f\left(v_{r}\right), v_{r+1}\right) \in$ $E(D)-E\left(\mathcal{C}_{D}\right)$ for each $r=1, \ldots, s-1$; every vertex $v_{1} \in V(D)$ forms the cyclic sequence $\left(v_{1}\right)$ from $v_{1}$ to $f\left(v_{1}\right)$, which is the case $s=1$. If a cyclic sequence $\left(v_{1}, \ldots, v_{s}\right)$ 
exists, then

$$
\begin{aligned}
v_{1} \rightarrow n\left(v_{1}\right) \rightarrow \cdots \rightarrow f\left(v_{1}\right) \rightarrow v_{2} & \rightarrow n\left(v_{2}\right) \rightarrow \\
& \cdots \rightarrow f\left(v_{2}\right) \rightarrow \cdots \rightarrow v_{s} \rightarrow n\left(v_{s}\right) \rightarrow \cdots \rightarrow f\left(v_{s}\right)
\end{aligned}
$$

is the associated walk from $v_{1}$ to $f\left(v_{s}\right)$. Note that the arcs between the consecutive vertices $v_{r}, n\left(v_{r}\right), \ldots, f\left(v_{r}\right)$ are in $\mathcal{C}_{D}\left(v_{r}\right)$ for $r=1, \ldots, s$. The digraph in Figure 2.1 contains the cyclic sequence $\left(v_{1}, v_{2}, v_{3}\right)$ from $v_{1}$ to $f\left(v_{3}\right)$. The associated walk from $v_{1}$ to $f\left(v_{3}\right)$ is

$$
v_{1} \rightarrow n\left(v_{1}\right) \rightarrow f\left(v_{1}\right) \rightarrow v_{2} \rightarrow n\left(v_{2}\right) \rightarrow f\left(v_{2}\right) \rightarrow v_{3} \rightarrow n\left(v_{3}\right) \rightarrow f\left(v_{3}\right) .
$$

Let $A=\left[a_{i j}\right]$ be an $m \times m$ matrix (over some field) with precisely one nonzero diagonal $\left\{a_{i \sigma(i)}\right\}$, where $\sigma$ is some permutation of $\{1, \ldots, m\}$ and all $a_{i \sigma(i)} \neq 0$. All such $A$ are necessarily nonsingular, and the digraph $D(A)$ with vertices $1, \ldots, m$ and $\operatorname{arcs}\left\{(i, j) \mid a_{i j} \neq 0\right\}$ belongs to the family $\mathcal{D}_{1}$. Thus, $\sigma$ is the permutation determined by $\mathcal{C}_{D(A)}$. Let $P=\left[p_{i j}\right]$ be the permutation matrix whose nonzero entries are in the same positions as the entries of the diagonal of $A$, and let $P^{-1}=\left[\pi_{i j}\right]$, i.e., with $n:=\sigma$,

$$
p_{i j}=\left\{\begin{array}{ll}
1 & , \text { if } j=n(i) ; \\
0 & , \text { otherwise }
\end{array} \quad \text { and } \quad \pi_{i j}= \begin{cases}1 & , \text { if } i=n(j) \\
0 & , \text { otherwise }\end{cases}\right.
$$

Throughout this section, we define $A^{\prime}=P^{-1} A=\left[a_{i j}^{\prime}\right]$, and let $A^{-1}=\left[\alpha_{i j}\right]$ and $\left(A^{\prime}\right)^{-1}=\left[\alpha_{i j}^{\prime}\right]$.

Lemma 2.1. Let $n:=\sigma$ and $f:=\sigma^{-1}$. Then

$$
a_{i j}=a_{n(i) j}^{\prime}, \quad \alpha_{i j}=\alpha_{i n(j)}^{\prime}, \quad a_{i j}^{\prime}=a_{f(i) j}, \quad \text { and } \quad \alpha_{i j}^{\prime}=\alpha_{i f(j)} .
$$

Proof. It follows from $A=P A^{\prime}$ that $a_{i j}=\sum_{k} p_{i k} a_{k j}^{\prime}=p_{i n(i)} a_{n(i) j}^{\prime}=a_{n(i) j}^{\prime}$. Since $A^{-1}=\left(A^{\prime}\right)^{-1} P^{-1}, \alpha_{i j}=\sum_{k} \alpha_{i k}^{\prime} \pi_{k j}=\alpha_{i n(j)}^{\prime} \pi_{n(j) j}=\alpha_{i n(j)}^{\prime}$. The third and fourth identities restate the first two statements. $\square$

Lemma 2.1 implies that $a_{i i}^{\prime}=a_{f(i) i} \neq 0$. The matrix $A^{\prime}$ therefore has only nonzero entries along its main diagonal. Since $A$ has only one nonzero diagonal, so does $A^{\prime}$, and $\mathcal{C}_{D\left(A^{\prime}\right)}$ consists of $m$ loops. Let $f^{\prime}$ be the "former" permutation in $D\left(A^{\prime}\right)$.

LEMma 2.2. Let $A$ be a matrix with precisely one nonzero diagonal, and let $P$ be a permutation matrix such that $A^{\prime}=P^{-1} A$ has nonzero main diagonal. Then the following three statements are equivalent:

1. $v_{1} \rightarrow \cdots \rightarrow v_{s}$ is a path in $D\left(A^{\prime}\right)$ from $v_{1}$ to $v_{s}$;

2. $\left(v_{1}, \ldots, v_{s}\right)$ is a cyclic sequence in $D\left(A^{\prime}\right)$ from $v_{1}$ to $f^{\prime}\left(v_{s}\right)=v_{s}$;

3. $\left(v_{1}, \ldots, v_{s}\right)$ is a cyclic sequence in $D(A)$ from $v_{1}$ to $f\left(v_{s}\right)$.

Proof. If $s=1$, then each statement is trivially true; so suppose for the remainder of the proof that $s \geq 2$. Since the factor $\mathcal{C}_{D\left(A^{\prime}\right)}$ contains only loops, it follows 
that $f^{\prime}(v)=v$ for all $v \in V\left(D\left(A^{\prime}\right)\right)$. Thus, if $v_{1} \rightarrow \ldots \rightarrow v_{s}$ is a path in $D\left(A^{\prime}\right)$ from $v_{1}$ to $v_{s}$, then $\left(f^{\prime}\left(v_{r}\right), v_{r+1}\right)=\left(v_{r}, v_{r+1}\right)$ is an arc in $E\left(D\left(A^{\prime}\right)\right)-E\left(\mathcal{C}_{D\left(A^{\prime}\right)}\right)$ for each $r=1, \ldots, s-1$. Hence, $\left(v_{1}, \ldots, v_{s}\right)$ is a cyclic sequence in $D\left(A^{\prime}\right)$ from $v_{1}$ to $v_{s}$. Conversely, if statement 2 is true, then the vertices $v_{1}, \ldots, v_{s}$ are distinct, and $\left(v_{r}, v_{r+1}\right)=\left(f^{\prime}\left(v_{r}\right), v_{r+1}\right)$ is an arc of $D\left(A^{\prime}\right)$ for all $r=1, \ldots, s-1$, so statement 1 is also true. Hence, statements 1 and 2 are equivalent.

Suppose that $\left(v_{1}, \ldots, v_{s}\right)$ is a cyclic sequence in $D\left(A^{\prime}\right)$. Then $v_{1}, \ldots, v_{s}$ are distinct, and $\left(v_{r}, v_{r+1}\right) \in E\left(D\left(A^{\prime}\right)\right)-E\left(\mathcal{C}_{D\left(A^{\prime}\right)}\right)$ for all $r=1, \ldots, s-1$. Lemma 2.1 implies that $a_{f\left(v_{r}\right) v_{r+1}}=a_{v_{r} v_{r+1}}^{\prime}$. Since in the latter equation, the right side is a nonzero entry of $A^{\prime}$ that is not on the unique nonzero diagonal of $A^{\prime}$, the left side must also be a nonzero entry of $A$ that is not on the unique nonzero diagonal of $A$. In other words, $\left(f\left(v_{r}\right), v_{r+1}\right) \in E(D(A))-E\left(\mathcal{C}_{D(A)}\right)$ for all $r=1, \ldots, s-1$, which proves that statement 2 implies statement 3 . The proof of the converse implication is similar and straightforward. $\square$

LEMMA 2.3. If $i=n(j)$ (i.e., $j=f(i)$ ), then $\alpha_{i j}=\frac{1}{a_{f(i) i}}=\frac{1}{a_{j i}}$.

Proof. By the identity $A^{-1}=\frac{\operatorname{adj} A}{\operatorname{det} A}$ and Lemma 2.1, $\alpha_{i j}=\alpha_{i f(i)}=\alpha_{i i}^{\prime}=\frac{\operatorname{det} A_{i i}^{\prime}}{\operatorname{det} A^{\prime}}$, where $A_{i i}^{\prime}$ is the matrix obtained by deleting the $i$ th row and the $i$ th column of the matrix $A^{\prime}$. Hence, $\alpha_{i j}=\frac{1}{a_{i i}^{\prime}}$, since $\operatorname{det} A_{i i}^{\prime}=\left(a_{11}^{\prime} \cdots a_{m m}^{\prime}\right) / a_{i i}^{\prime}$ and $\operatorname{det} A^{\prime}=$ $a_{11}^{\prime} \cdots a_{m m}^{\prime}$. By Lemma 2.1, $\alpha_{i j}=\frac{1}{a_{f(i) i}}$.

Suppose that $i \neq n(j)$. It follows from [12, Corollary 9.1] that

$$
\alpha_{i n(j)}^{\prime}=\frac{1}{\operatorname{det} A^{\prime}} \sum_{p_{k}}(-1)^{l_{k}} \operatorname{det} A^{\prime}\left[V\left(D\left(A^{\prime}\right)\right)-V\left(p_{k}\right)\right] \prod_{(u, v) \in E\left(p_{k}\right)} a_{u v}^{\prime}
$$

where the sum is taken over all paths $p_{k}$ in $D\left(A^{\prime}\right)$ from $i$ to $n(j)$, where $l_{k}$ is the length of path $p_{k}$, where $V\left(p_{k}\right)$ (resp. $E\left(p_{k}\right)$ ) is the set of vertices (resp. arcs) of path $p_{k}$, and $A^{\prime}\left[V\left(D\left(A^{\prime}\right)\right)-V\left(p_{k}\right)\right]$ is the submatrix of $A^{\prime}$ containing rows and columns corresponding to the vertices not in $V\left(p_{k}\right)$. Thus,

$$
\alpha_{i n(j)}^{\prime}=\sum_{p_{k}}(-1)^{l_{k}} \frac{\prod_{(u, v) \in E\left(p_{k}\right)} a_{u v}^{\prime}}{\prod_{q \in V\left(p_{k}\right)} a_{q q}^{\prime}},
$$

since $\operatorname{det} A^{\prime}=a_{11}^{\prime} \cdots a_{m m}^{\prime}$. By Lemma 2.2,

$$
\alpha_{i n(j)}^{\prime}=\sum_{\mathbf{v}}(-1)^{s-1} \frac{\prod_{r=1}^{s-1} a_{v_{r} v_{r+1}}^{\prime}}{\prod_{q=1}^{s} a_{v_{q} v_{q}}^{\prime}},
$$

where the sum is taken over all cyclic sequences $\mathbf{v}=\left(v_{1}, \ldots, v_{s}\right)$ in $D\left(A^{\prime}\right)$ from $v_{1}=i$ to $f^{\prime}\left(v_{s}\right)=v_{s}=n(j)$, and $s=|\mathbf{v}|$ is the length of sequence $\mathbf{v}$. Lemma 2.1 implies 
that

$$
\alpha_{i j}=\alpha_{i n(j)}^{\prime}=\sum_{\mathbf{v}}(-1)^{s-1} \frac{a_{f\left(v_{1}\right) v_{2}} \cdots a_{f\left(v_{s-1}\right) v_{s}}}{a_{f\left(v_{1}\right) v_{1}} a_{f\left(v_{2}\right) v_{2}} \cdots a_{f\left(v_{s}\right) v_{s}}}
$$

where the sum is taken over all cyclic sequences $\mathbf{v}=\left(v_{1}, \ldots, v_{s}\right)$ in $D(A)$ from $v_{1}=i$ to $f\left(v_{s}\right)=j$, and $s=|\mathbf{v}|$.

If $i=n(j)$, then $(i)$ is the only cyclic sequence from $i$ to $j=f(i)$ in $D(A)$. Thus, Lemma 2.3 and the calculations above together imply the following theorem.

TheOREM 2.4. Let $A$ be a square matrix with precisely one nonzero diagonal, and let $A^{-1}=\left[\alpha_{i j}\right]$. Then, for all $i, j$,

$$
\alpha_{i j}=\sum_{\mathbf{v}}(-1)^{s-1} \frac{a_{f\left(v_{1}\right) v_{2}} \cdots a_{f\left(v_{s-1}\right) v_{s}}}{a_{f\left(v_{1}\right) v_{1}} a_{f\left(v_{2}\right) v_{2}} \cdots a_{f\left(v_{s}\right) v_{s}}}
$$

where the sum is taken over all cyclic sequences $\mathbf{v}=\left(v_{1}, \ldots, v_{s}\right)$ in $D(A)$ from $i=v_{1}$ to $j=f\left(v_{s}\right)$, and $s=|\mathbf{v}|$ is the length of sequence $\mathbf{v}$. If no such sequence exists, then $\alpha_{i j}=0$.

ExAmple 2.5. To illustrate Theorem 2.4, a matrix $A$ with precisely one nonzero diagonal, the digraph $D=D(A)$, and the inverse matrix $A^{-1}$ are displayed in Figure 2.2. Since $\mathcal{C}_{D}$ consists of the loop $(1,1)$ and the 2 -cycle $2 \rightarrow 3 \rightarrow 2$, the permutation $f$ in $D$ satisfies $f(1)=1, f(2)=3$, and $f(3)=2$. To compute the entry $\alpha_{33}$ of $A^{-1}$, for instance, first find all cyclic sequences in $D(A)$ from $i=3$ to $j=3=f(2)$. These are the sequences $(3,2)$ of length 2 and $(3,1,2)$ of length 3 , with associated walks $3 \rightarrow 2 \rightarrow 2 \rightarrow 3$ and $3 \rightarrow 2 \rightarrow 1 \rightarrow 1 \rightarrow 2 \rightarrow 3$, respectively. Theorem 2.4 then implies that the entry $\alpha_{33}$ equals

$$
(-1)^{2-1} \frac{a_{f(3) 2}}{a_{f(3) 3} a_{f(2) 2}}+(-1)^{3-1} \frac{a_{f(3) 1} a_{f(1) 2}}{a_{f(3) 3} a_{f(1) 1} a_{f(2) 2}}=-\frac{a_{22}}{a_{23} a_{32}}+\frac{a_{21} a_{12}}{a_{23} a_{11} a_{32}} .
$$

$\left[\begin{array}{ccc}a_{11} & a_{12} & 0 \\ a_{21} & a_{22} & a_{23} \\ 0 & a_{32} & 0\end{array}\right]$

$A$

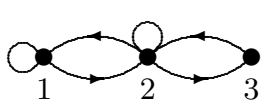

$D(A)$

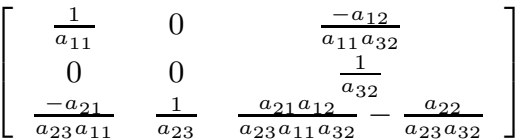

$A^{-1}$

FIG. 2.2. Matrices and digraph for Example 2.5

3. Digraphs and matrix inversion. If $D(A) \in \mathcal{D}_{1}$, then there exist permutation matrices $P, Q$ such that $P A Q$ is triangular; thus $D\left(A^{-1}\right) \in \mathcal{D}_{1}$. However, the actual form of $D\left(A^{-1}\right)$ is not necessarily determined by $D$. In other words, if $D$ is the digraph of matrices $A_{1}$ and $A_{2}$, then $D\left(A_{1}^{-1}\right)$ and $D\left(A_{2}^{-1}\right)$ are not necessarily 
identical. To illustrate this, consider real matrices with digraphs as in Figure 2.2. If, for instance,

$$
A_{1}=\left[\begin{array}{ccc}
-1 & 1 & 0 \\
1 & 1 & -1 \\
0 & -1 & 0
\end{array}\right], \quad \text { then } \quad A_{1}^{-1}=\left[\begin{array}{ccc}
-1 & 0 & -1 \\
0 & 0 & -1 \\
-1 & -1 & -2
\end{array}\right]
$$

and the digraph $D\left(A_{1}^{-1}\right)$ contains the loop $(3,3)$. If, on the other hand,

$$
A_{2}=\left[\begin{array}{ccc}
-1 & -1 & 0 \\
1 & 1 & -1 \\
0 & -1 & 0
\end{array}\right], \text { then } A_{2}^{-1}=\left[\begin{array}{ccc}
-1 & 0 & 1 \\
0 & 0 & -1 \\
-1 & -1 & 0
\end{array}\right]
$$

and $D\left(A_{2}^{-1}\right)$ has no $(3,3)$ loop.

The following two examples show that for any digraph $D \in \mathcal{D}_{1}$ with at least two cyclic sequences between some pair of vertices, there exist matrices $A_{1}, A_{2}$ with $D\left(A_{1}\right)=D\left(A_{2}\right)=D$ such that $D\left(A_{1}^{-1}\right)$ is not equal to $D\left(A_{2}^{-1}\right)$.

ExAmple 3.1. Let $D \in \mathcal{D}_{1}$ and let $A_{1}=\left[a_{u v}^{(1)}\right]$ be the real matrix with digraph $D\left(A_{1}\right)=D$ and entries

$$
a_{u v}^{(1)}=\left\{\begin{array}{cl}
-1 & , \text { if }(u, v) \in E\left(\mathcal{C}_{D}\right) \\
1 & , \text { if }(u, v) \in E(D)-E\left(\mathcal{C}_{D}\right) \\
0 & , \text { if }(u, v) \notin E(D) .
\end{array}\right.
$$

For fixed vertices $i$ and $j$, suppose that there are $x \geq 1$ cyclic sequences from $i$ to $j$. Let $A_{1}^{-1}=\left[\alpha_{u v}^{(1)}\right]$. By Theorem 2.4, the contribution to the entry $\alpha_{i j}^{(1)}$ from each cyclic sequence $\left(v_{1}, \ldots, v_{s}\right)$ from $v_{1}=i$ to $f\left(v_{s}\right)=j$ is $(-1)^{s-1} \frac{1^{s-1}}{(-1)^{s}}=-1$. Hence, $\alpha_{i j}^{(1)}=-x \neq 0$, and $D\left(A_{1}^{-1}\right)$ contains the $\operatorname{arc}(i, j)$.

EXAMPLE 3.2. Let $D \in \mathcal{D}_{1}$. For fixed vertices $i$ and $j$, suppose that there are $y \geq 2$ cyclic sequences from $i$ to $j$ of the form $\left(v_{1}, \ldots, v_{s}\right)$. Note that there must exist an $\operatorname{arc}(p, q) \in E(D)-E\left(\mathcal{C}_{D}\right)$ that is not contained in every set of arcs $\left\{\left(f\left(v_{r}\right), v_{r+1}\right) \mid r=1, \ldots, s-1\right\}$ associated with these cyclic sequences. Suppose that $(p, q)$ is contained in $z(1 \leq z \leq y-1)$ such sets of arcs. Let $A_{2}=\left[a_{u v}^{(2)}\right]$ be equal to the matrix $A_{1}$ in Example 3.1, except that $a_{p q}^{(2)}=\frac{z-y}{z}$, and let $A_{2}^{-1}=\left[\alpha_{u v}^{(2)}\right]$. By Theorem 2.4, $y-z$ cyclic sequences from $i$ to $j$ each contribute -1 to the entry $\alpha_{i j}^{(2)}$, whereas the remaining $z$ cyclic sequences from $i$ to $j$ each contribute $-\left(\frac{z-y}{z}\right)$. Hence, $\alpha_{i j}^{(2)}=-(y-z)+z\left(\frac{y-z}{z}\right)=0$, and $(i, j) \notin D\left(A_{2}^{-1}\right)$.

In contrast with the above examples, we now give a necessary and sufficient condition for which $D\left(A^{-1}\right)$ is uniquely determined by $D(A)$.

ThEOREM 3.3. Let $D \in \mathcal{D}_{1}$. Then the digraph $D\left(A^{-1}\right)$ is the same for all matrices $A$ that have digraph $D=D(A)$ if and only if there is at most one cyclic sequence between each pair of vertices.

Proof. If no cyclic sequence exists between vertices $i$ and $j$, then Theorem 2.4 states that $\alpha_{i j}=0$, so $(i, j)$ is not an arc of $D\left(A^{-1}\right)$. If there is precisely one 
cyclic sequence between $i$ and $j$, then Theorem 2.4 implies that $\alpha_{i j} \neq 0$, so $D\left(A^{-1}\right)$ contains the arc $(i, j)$. Thus, if there is at most one cyclic sequence between each pair of vertices $i$ and $j$, then the digraph $D\left(A^{-1}\right)$ is the same for all matrices $A$ that have digraph $D=D(A)$.

Conversely, for fixed vertices $i$ and $j$, suppose that there are $x \geq 2$ cyclic sequences from $i$ to $j$. Let $A_{1}$ and $A_{2}$ be the matrices with digraphs $D\left(A_{1}\right)=D\left(A_{2}\right)=D$ defined in Examples 3.1 and 3.2, respectively. Then $D\left(A_{1}^{-1}\right)$ contains the arc $(i, j)$, whereas $D\left(A_{2}^{-1}\right)$ does not. Therefore, $D\left(A_{1}^{-1}\right) \neq D\left(A_{2}^{-1}\right)$.

A digraph $D$ is unipathic [11] if there is at most one path in $D$ between any pair of distinct vertices. The following result is an immediate consequence of Lemmas 2.2 and 2.3, and Theorem 3.3.

Corollary 3.4. Let $D \in \mathcal{D}_{1}$ be a digraph such that $\mathcal{C}_{D}=\{(v, v) \mid v \in V(D)\}$. Then $D\left(A^{-1}\right)$ is independent of the particular matrix $A$ with $D(A)=D$ if and only if $D$ is unipathic.

4. Sign patterns and matrix inversion. This section is restricted to matrices over the real field. For any real $m \times m$ matrix $A=\left[a_{i j}\right]$, let the sign pattern $\mathcal{Q}(A)$ denote the family of real $m \times m$ matrices $B=\left[b_{i j}\right]$ where each entry $b_{i j}$ has the same sign $\{-, 0,+\}$ as $a_{i j}$. A real matrix $A$ is sign-nonsingular [4, Section 1.2] if each $B \in \mathcal{Q}(A)$ is nonsingular; thus if $D(A) \in \mathcal{D}_{1}$, then $A$ is sign-nonsingular. A matrix $A$ is strongly sign-nonsingular [4, Chapter 7$]$ if it is sign-nonsingular and $B^{-1} \in \mathcal{Q}\left(A^{-1}\right)$ for each matrix $B \in \mathcal{Q}(A)$.

Example 3.1 demonstrates that for each digraph $D \in \mathcal{D}_{1}$ there exists at least one strongly sign-nonsingular matrix $A_{1}$ such that $D\left(A_{1}\right)=D$. Conversely, if $D \in \mathcal{D}_{1}$ with vertices $i$ and $j$ between which there are at least two cyclic sequences, then there is at least one matrix $A_{2}$ with $D\left(A_{2}\right)=D$ that is not strongly sign-nonsingular, as illustrated by Example 3.2.

The following result gives necessary and sufficient conditions for strong signnonsingularity.

Proposition 4.1. [4, Corollary 3.2.3] Let $A=\left[a_{i j}\right]$ be a real square matrix with a negative main diagonal. Then $A$ is strongly sign-nonsingular if and only if

1. for each cycle $i_{1} \rightarrow i_{2} \rightarrow \cdots \rightarrow i_{k} \rightarrow i_{1}$ in $D(A), a_{i_{1} i_{2}} \cdots a_{i_{k} i_{1}}<0$, and

2. for all $i \neq j$, the sign of the product $a_{v_{1} v_{2}} \cdots a_{v_{s-1} v_{s}}$ is the same for all paths $v_{1} \rightarrow \cdots \rightarrow v_{s}$ in $D(A)$ from $i$ to $j$.

The next lemma expresses Proposition 4.1 in terms of cyclic sequences for digraphs in $\mathcal{D}_{1}$. This can be proved by using Lemma 2.2 and the fact that strong sign-nonsingularity is invariant with respect to row permutations.

Lemma 4.2. Let $A$ be a real matrix with $D=D(A) \in \mathcal{D}_{1}$. If each entry $a_{i j}$ with $(i, j) \in E\left(\mathcal{C}_{D}\right)$ has negative sign, then $A$ is strongly sign-nonsingular if and only if for all $i, j$, the sign of the product $a_{f\left(v_{1}\right) v_{2}} \cdots a_{f\left(v_{s-1}\right) v_{s}}$ is the same for all cyclic sequences $\mathbf{v}=\left(v_{1}, \ldots, v_{s}\right)$ in $D(A)$ from $v_{1}=i$ to $f\left(v_{s}\right)=j$.

Theorem 4.3 below extends Theorem 3.3 and Corollary 3.4 .

THEOREM 4.3. If $D$ is a digraph on $m$ vertices, then the following statements are equivalent:

1. For each real matrix $A$ with $D(A)=D, A$ is strongly sign-nonsingular; 
2. For each matrix $A$ with $D(A)=D, A$ is nonsingular and $D\left(A^{-1}\right)$ is uniquely determined;

3. $D \in \mathcal{D}_{1}$ and there is at most one cyclic sequence in $D$ between any pair of vertices;

4. For each matrix $A$ with $D(A)=D, A$ contains precisely one nonzero diagonal, and each order $m-1$ square submatrix of $A$ has at most one nonzero diagonal.

Furthermore, if $D$ has a loop at each vertex, then the statements above are equivalent to the following statement:

5. $D \in \mathcal{D}_{1}$ and $D$ is unipathic.

Proof. Each of the conditions in statements 1-4 is invariant under row permutations and implies that $D$ has at least one factor, so without loss of generality assume that $D$ has a loop at each vertex.

We first prove that statements 1, 2, 3, and 5 are equivalent. If statement 5 is false, then $D$ contains either a cycle of length greater than or equal to two or a pair of distinct vertices between which there are at least two distinct paths. Then it is possible to choose a matrix $A$ with $D(A)=D$ such that at least one of the conditions in Proposition 4.1 is not satisfied, so $A$ is not strongly sign-nonsingular. Thus by taking the contrapositive, statement 1 implies statement 5 .

If statement 5 is true, then $D \in \mathcal{D}_{1}$ and there is at most one path in $D$ between any pair of distinct vertices. Thus by Lemma 2.2 , since $D$ has a loop at each vertex, there is at most one cyclic sequence in $D$ between any pair of distinct vertices. Hence, statement 5 implies statement 3.

Assume that statement 3 is true and that $A$ is a matrix with $D(A)=D$. By Theorem 2.4, $(i, j) \in E\left(D\left(A^{-1}\right)\right)$ if and only if there exists a cyclic sequence in $D$ from $i$ to $j$. This uniquely determines $D\left(A^{-1}\right)$, so statement 3 implies statement 2 .

If there exists a matrix $A$ with $D(A)=D$ that is singular, then statements 1 and 2 are both false. Therefore, assume that for each matrix $A$ with $D(A)=D$, $A$ is nonsingular, and assume also that statement 1 is false. Then there exists a real matrix $A$ with $D(A)=D$ that is not strongly sign-nonsingular. Thus, there exists a matrix $B$ with the same sign pattern as $A$ such that $A^{-1}$ and $B^{-1}$ do not have the same sign pattern. If $D\left(A^{-1}\right) \neq D\left(B^{-1}\right)$, then statement 2 is false. If $D\left(A^{-1}\right)=D\left(B^{-1}\right)$, then let $i, j$ be indices such that the entries $\left(A^{-1}\right)_{i j}$ and $\left(B^{-1}\right)_{i j}$ are opposite in sign, say $\left(A^{-1}\right)_{i j}<0$ and $\left(B^{-1}\right)_{i j}>0$. The set $\mathcal{Q}(A)$ is connected and each matrix $X \in \mathcal{Q}(A)$ is nonsingular by assumption, since $D(X)=D$. Thus, the map $g: \mathcal{A} \mapsto \mathbb{R}$ given by $g(X)=\left(X^{-1}\right)_{i j}$ is well-defined. Since $g$ is continuous, the image $g(\mathcal{Q}(A))$ must be connected, i.e., $g(\mathcal{Q}(A))$ is a real interval. Thus, since $g(A)<0$ and $g(B)>0$, there exists a matrix $X \in \mathcal{Q}(A)$ with $g(X)=0$. Then $D\left(X^{-1}\right) \neq D\left(A^{-1}\right)$, so statement 2 is false. By taking the contrapositive, statement 2 implies statement 1 . Hence, statements 1, 2, 3, and 5 are equivalent.

To prove that statements 4 and 5 are equivalent, suppose that $D \in \mathcal{D}_{1}$, and let $A$ be a matrix with $D(A)=D$. Thus, each order $m-1$ principal submatrix of $A$ contains precisely one nonzero diagonal, namely the main diagonal. For all $i \neq j$, consider the submatrix $A_{j i}$ of $A$ with row $j$ and column $i$ deleted. Then 
$i \rightarrow i_{1} \rightarrow i_{2} \rightarrow \ldots \rightarrow i_{k} \rightarrow j$ is a path in $D$ if and only if

$$
\left\{a_{k k} \mid k \neq i, i_{1}, i_{2}, \ldots, i_{k}, j\right\} \cup\left\{a_{i i_{1}}, a_{i_{1} i_{2}}, \ldots, a_{i_{k} j}\right\}
$$

is a nonzero diagonal of $A_{j i}$. Hence, statements 4 and 5 are equivalent.

5. Nonsingular nearly reducible matrices. A digraph $D$ is said to be minimally strongly connected if it is strongly connected and the removal of any arc of $D$ causes the digraph to no longer be strongly connected. In terms of matrices (over some field), minimally strongly connected digraphs correspond to nearly reducible matrices, i.e., irreducible matrices that become reducible whenever any nonzero entry is replaced by zero. More precisely, a square matrix $A$ is nearly reducible [3, Section 3.3] if and only if its digraph $D(A)$ is minimally strongly connected.

Minimally strongly connected digraphs may be characterized in several ways. One such way is presented in the following lemma. For other characterizations, see $[2,5$, $8,10]$. A digraph $D$ is arc unique [9] if for each $\operatorname{arc}(u, v)$ of $D, u \rightarrow v$ is the only path in $D$ between $u$ and $v$. Note that a strongly connected arc unique digraph with at least two vertices cannot contain loops, and that all unipathic digraphs without loops are arc unique.

Lemma 5.1. [9] A digraph on at least two vertices is minimally strongly connected if and only if it is a strongly connected arc unique digraph.

Proof. Let $D$ be a strongly connected digraph on at least two vertices. Suppose that $D$ is minimally strongly connected and let $(u, v)$ be an arc of $D$. By the minimality of $D, u \neq v$ and $u \rightarrow v$ is the only path in $D$ from $u$ to $v$, so $D$ is arc unique. Conversely, suppose that $D$ is arc unique and let $(u, v)$ be an arc of $D$. Since $u \neq v$ and $u \rightarrow v$ is the only path in $D$ from $u$ to $v$, the digraph $D-(u, v)$ is not strongly connected. Thus, $D$ is minimally strongly connected.

Hedrick and Sinkhorn [7] proved that the permanent of a nearly reducible matrix contains at most one term. Restated in terms of digraphs, this result may be expressed as follows.

TheOREM 5.2. [7] A minimally strongly connected digraph D contains at most one factor.

We now focus on the class of minimally strongly connected digraphs $D$ with one factor (i.e., $D \in \mathcal{D}_{1}$ ), and in particular on properties of their cyclic sequences. We then use results of Section 2 to determine in Theorem 5.8 the entries of the inverse of a nonsingular nearly reducible matrix. The result of the following lemma can also be found in [3, p. 63].

LEMMA 5.3. Let $C$ be a closed walk in a minimally strongly connected digraph $D$, and let $u$ and $v$ be distinct vertices of $C$. If $(u, v)$ is an arc of $D$, then $(u, v)$ is an arc of $C$. In other words, $C$ has no chords.

Proof. If $(u, v)$ is an arc of $D$ not contained in $C$, then $C$ and therefore $D$ contains a walk (and thus a path) from $u$ to $v$ that does not contain the $\operatorname{arc}(u, v)$. But this contradicts the arc uniqueness of $D$.

LEMma 5.4. If $D \in \mathcal{D}_{1}$ is a minimally strongly connected digraph, then all the cycles $\mathcal{C}_{D}\left(v_{1}\right), \ldots, \mathcal{C}_{D}\left(v_{s}\right)$ associated with a cyclic sequence $\left(v_{1}, \ldots, v_{s}\right)$ are distinct. In particular, the walk associated with any cyclic sequence in $D$ is a path. 
Proof. Suppose $q<r$ are indices of $\{1, \ldots, s\}$ such that $\mathcal{C}_{D}\left(v_{q}\right)$ and $\mathcal{C}_{D}\left(v_{r}\right)$ are identical and $r$ is minimal with respect to this property. By definition, $v_{1}, \ldots, v_{s}$ are distinct and $\left(f\left(v_{q}\right), v_{q+1}\right) \in E(D)-E\left(\mathcal{C}_{D}\right)$. If $\mathcal{C}_{D}\left(v_{q+1}\right)=\mathcal{C}_{D}\left(v_{q}\right)$, then $\left(f\left(v_{q}\right), v_{q+1}\right)$ is a chord of $\mathcal{C}_{D}\left(v_{q}\right)$, which contradicts Lemma 5.3. Thus, $\mathcal{C}_{D}\left(v_{q+1}\right) \neq \mathcal{C}_{D}\left(v_{q}\right)$. If $r \geq q+2$, then the minimality of $r$ implies that

$$
f\left(v_{q}\right) \rightarrow v_{q+1} \rightarrow n\left(v_{q+1}\right) \rightarrow \cdots \rightarrow f\left(v_{q+1}\right) \rightarrow \cdots \rightarrow v_{r} \rightarrow n\left(v_{r}\right) \rightarrow \cdots \rightarrow f\left(v_{q}\right)
$$

is a closed walk with the chord $\left(f\left(v_{q+1}\right), v_{q+1}\right)$, which contradicts Lemma 5.3. The cycles $\mathcal{C}_{D}\left(v_{1}\right), \ldots, \mathcal{C}_{D}\left(v_{s}\right)$ are therefore distinct.

Corollary 5.5. Let $D \in \mathcal{D}_{1}$ be a minimally strongly connected digraph. If $i$ and $j \neq f(i)$ are vertices contained in the same cycle $C$ of $\mathcal{C}_{D}$, then there is no cyclic sequence from $i$ to $j$. In particular, there is no cyclic sequence from a vertex to itself.

Proof. If $j \neq f(i)$, then $(i)$ is not a cyclic sequence from $i$ to $j$. Therefore, if $\left(v_{1}, \ldots, v_{s}\right)$ is a cyclic sequence from $v_{1}=i$ to $f\left(v_{s}\right)=j$, then it must have length $s \geq 2$, and $i \notin \mathcal{C}_{D}\left(v_{s}\right)=\mathcal{C}_{D}\left(f\left(v_{s}\right)\right)=\mathcal{C}_{D}(j)$, by Lemma 5.4. The second statement follows from the fact that a minimally strongly connected digraph has no loops, giving that $i \neq f(i)$ for all vertices $i$.

Lemma 5.6. If $D \in \mathcal{D}_{1}$ is a minimally strongly connected digraph, then there is at most one cyclic sequence between any two vertices of $D$.

Proof. Suppose that $\left(v_{1}, \ldots, v_{s}\right)$ and $\left(w_{1}, \ldots, w_{t}\right)$ are distinct cyclic sequences from $i$ to $j$. Since $v_{1}=w_{1}(=i)$, there is a maximal index $q<\min \{s, t\}$ such that $v_{q}=w_{q}$ and $v_{q+1} \neq w_{q+1}$. Now, $f\left(v_{s}\right)=f\left(w_{t}\right)(=j)$, so $v_{s}=w_{t}$. Hence, there are minimal indices $r, r^{\prime}>q$ such that $\mathcal{C}_{D}\left(v_{r}\right)=\mathcal{C}_{D}\left(w_{r^{\prime}}\right)$. Assume that $r=q+1$. Then

$$
\begin{aligned}
f\left(w_{q}\right) & \rightarrow w_{q+1} \rightarrow n\left(w_{q+1}\right) \rightarrow \cdots \rightarrow f\left(w_{q+1}\right) \\
& \rightarrow w_{q+2} \rightarrow \cdots \rightarrow w_{r^{\prime}} \rightarrow n\left(w_{r^{\prime}}\right) \rightarrow \cdots \rightarrow v_{r}
\end{aligned}
$$

is a path from $f\left(v_{q}\right)=f\left(w_{q}\right)$ to $v_{r}=v_{q+1}$. Since $\left(f\left(v_{q}\right), v_{q+1}\right)$ is an arc of $D$, this contradicts the arc uniqueness of $D$. Hence, $r>q+1$. Similarly, $r^{\prime}>q+1$.

Thus, assume that $r, r^{\prime} \geq q+2$. Since $D$ is strongly connected, there is a path in $D$ from $n\left(v_{r}\right)$ to $v_{q+1}$. Lemma 5.3 implies that this path must contain the arc $\left(f\left(v_{q+1}\right), v_{q+1}\right)$; otherwise, this arc would be a chord of the closed walk

$$
v_{q+1} \rightarrow n\left(v_{q+1}\right) \rightarrow \cdots \rightarrow f\left(v_{q+1}\right) \rightarrow \cdots \rightarrow v_{r} \rightarrow \cdots \rightarrow v_{q+1} .
$$

However, this implies the existence of a walk

$$
f\left(v_{q}\right)=f\left(w_{q}\right) \rightarrow w_{q+1} \rightarrow \ldots \rightarrow w_{r^{\prime}} \rightarrow n\left(w_{r^{\prime}}\right) \rightarrow \cdots \rightarrow v_{r} \rightarrow \cdots \rightarrow f\left(v_{q+1}\right) \rightarrow v_{q+1}
$$

that does not contain the $\operatorname{arc}\left(f\left(v_{q}\right), v_{q+1}\right)$. This contradicts the arc uniqueness of $D$, since $D$ contains the $\operatorname{arc}\left(f\left(v_{q}\right), v_{q+1}\right)$. $\square$

The next result follows immediately from Theorem 5.2 and Lemmas 2.2 and 5.6.

Corollary 5.7. Let $A$ be a nonsingular nearly reducible matrix, and let $P$ be the permutation matrix such that the main diagonal of $A^{\prime}=P^{-1} A$ contains only nonzero entries. Then $D\left(A^{\prime}\right) \in \mathcal{D}_{1}$ is unipathic and arc unique (but not strongly connected). 
An explicit description of the inverse of a nonsingular nearly reducible matrix $A$, in terms of the digraph $D(A)$, now follows from Theorems 2.4 and 5.2, and Lemma 5.6.

THEOREM 5.8. Let $A=\left[a_{i j}\right]$ be a nonsingular nearly reducible matrix, and let $A^{-1}=\left[\alpha_{i j}\right]$. If there is a cyclic sequence $\left(v_{1}, \ldots, v_{s}\right)$ in $D(A)$ from vertex $i=v_{1}$ to vertex $j=f\left(v_{s}\right)$, then

$$
\alpha_{i j}=(-1)^{s-1} \frac{a_{f\left(v_{1}\right) v_{2}} \cdots a_{f\left(v_{s-1}\right) v_{s}}}{a_{f\left(v_{1}\right) v_{1}} a_{f\left(v_{2}\right) v_{2}} \cdots a_{f\left(v_{s}\right) v_{s}}} ;
$$

otherwise $\alpha_{i j}=0$.

Corollary 5.9. If $A$ is a nonsingular nearly reducible real matrix, then $A$ is strongly sign-nonsingular.

This corollary also follows from a more general result for the Moore-Penrose inverse of a nearly reducible matrix in [1]. The strongly sign-nonsingular matrix $A=\left[\begin{array}{ll}1 & 1 \\ 1 & 0\end{array}\right]$ shows that the converse of Corollary 5.9 does not hold since $A$ is not nearly reducible. Entries of the inverse corresponding to the particular cyclic sequences of length one or two in $D(A)$ in Theorem 5.8 have the following simple forms.

COROLlary 5.10. Let $A=\left[a_{i j}\right]$ be a nonsingular nearly reducible matrix with $a_{r t} \neq 0$ and let $A^{-1}=\left[\alpha_{i j}\right]$. If $a_{r t}$ lies on the unique nonzero diagonal of $A$ (i.e., $t=n(r))$, then $\alpha_{t r}=\frac{1}{a_{r t}}$; otherwise, $\alpha_{n(r) f(t)}=\frac{-a_{r t}}{a_{r n(r)} a_{f(t) t}}$. These cases correspond to the cyclic sequences $(n(r))$ and $(n(r), t)$ in $D(A)$, respectively.

Corollary 5.10 describes in detail key aspects of the inversion $A \mapsto A^{-1}$ of a nonsingular nearly reducible matrix $A$. In terms of the digraphs $D(A)$ and $D\left(A^{-1}\right)$, the map $D(A) \mapsto D\left(A^{-1}\right)$ replaces each arc $(r, t) \in E(D(A))$ by the arc $(n(r), f(t))$. If $(r, t) \in E\left(\mathcal{C}_{D(A)}\right)$, then this replacement merely reverses the direction of $(r, t)$. Thus, the factor $\mathcal{C}_{D\left(A^{-1}\right)}$ may be obtained by reversing the direction of the cycles in $\mathcal{C}_{D(A)}$. For any cycles $C_{1}, C_{2} \in \mathcal{C}_{D(A)}$, if $(r, t) \in E(D(A))$ is an arc connecting $C_{1}$ to $C_{2}$ (i.e., $\left.r \in V\left(C_{1}\right), t \in V\left(C_{2}\right)\right)$, then $(n(r), f(t)) \in E\left(D\left(A^{-1}\right)\right)$ is an arc connecting the corresponding reversed cycles in $D\left(A^{-1}\right)$. Hence, since $D(A)$ is strongly connected, $D\left(A^{-1}\right)$ is strongly connected. In addition to the arcs resulting from the arc replacements described above, $D\left(A^{-1}\right)$ may also contain arcs that result from cyclic sequences of length greater than two.

COROLlaRY 5.11. If $A$ is a nonsingular nearly reducible $m \times m$ matrix, then each order $m-1$ square submatrix of $A$ has at most one nonzero diagonal, and no order $m-1$ principal submatrix of $A$ has a nonzero diagonal. In particular, the order $m-1$ principal submatrices of $A$ are all singular.

Proof. If $A$ is a nonsingular nearly reducible $m \times m$ matrix, then this is equally true for each matrix $B$ with $D(B)=D(A)$. By Corollary 5.9, each such matrix $B$ is strongly sign-nonsingular, thus by Theorem 4.3 , each order $m-1$ square submatrix of $A$ has at most one nonzero diagonal. Corollary 5.5 and Theorem 5.8 together imply that the entries along the main diagonal of $A^{-1}$ are all zero. Thus, the identity $A^{-1}=\frac{\operatorname{adj} A}{\operatorname{det} A}$ implies that the order $m-1$ principal minors of $A$ must all be zero. The order $m-1$ principal submatrices of $A$ can therefore not have precisely one nonzero diagonal; thus they must contain none.

Note that by this corollary, none of the digraphs $D(A)-v, v \in V(D(A))$, has a factor. 
EXAMPLE 5.12. To illustrate some of the results above, a nearly reducible matrix $A=\left[a_{i j}\right]$, its inverse $A^{-1}=\left[\alpha_{i j}\right]$, and the associated digraphs $D(A)$ and $D\left(A^{-1}\right)$ are displayed in Figure 5.1. The factor $\mathcal{C}_{D(A)}$ consists of the 2-cycle $1 \rightarrow 2 \rightarrow 1$ and the 3 -cycle $3 \rightarrow 4 \rightarrow 5 \rightarrow 3$, and the permutations $n$ and $f$ in $D(A)$ satisfy $n(1)=2$, $n(2)=1, n(3)=4, n(4)=5, n(5)=3$, and $f=n^{-1}$. Note that $(1,2) \in E\left(\mathcal{C}_{D(A)}\right)$ whereas $(2,3) \in E(D(A))-E\left(\mathcal{C}_{D(A)}\right)$. Thus by Corollary 5.10 , the entry $a_{12}$ of $A$ is replaced in $A^{-1}$ by the entry $\alpha_{21}=\frac{1}{a_{12}}$, and the entry $a_{23}$ in $A$ is replaced in $A^{-1}$ by the entry $\alpha_{15}=\frac{-a_{23}}{a_{21} a_{53}}$. In terms of digraphs, the arcs $E\left(\mathcal{C}_{D\left(A^{-1}\right)}\right)$ are obtained by reversing the direction of each arc in $E\left(\mathcal{C}_{D(A)}\right)$, and the arcs $(2,3)$ and $(3,2)$ are replaced in $D\left(A^{-1}\right)$ by the arcs $(1,5)=(n(2), f(3))$ and $(4,1)=(n(3), f(2))$, respectively. Note that if $A$ is real, then $A$ is strongly sign-nonsingular.

$$
\left[\begin{array}{ccccc}
0 & a_{12} & 0 & 0 & 0 \\
a_{21} & 0 & a_{23} & 0 & 0 \\
0 & a_{32} & 0 & a_{34} & 0 \\
0 & 0 & 0 & 0 & a_{45} \\
0 & 0 & a_{53} & 0 & 0
\end{array}\right]
$$

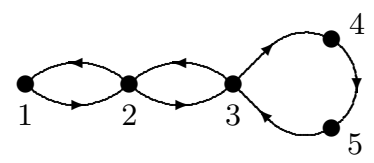

$D(A)$

$$
\left[\begin{array}{ccccc}
0 & \frac{1}{a_{21}} & 0 & 0 & \frac{-a_{23}}{a_{21} a_{53}} \\
\frac{1}{a_{12}} & 0 & 0 & 0 & 0 \\
0 & 0 & 0 & 0 & \frac{1}{a_{53}} \\
\frac{-a_{32}}{a_{12} a_{34}} & 0 & \frac{1}{a_{34}} & 0 & 0 \\
0 & 0 & 0 & \frac{1}{a_{45}} & 0
\end{array}\right]
$$

$$
A^{-1}
$$

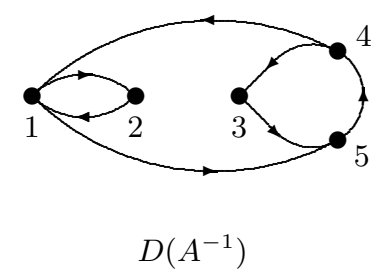

FIG. 5.1. Matrices and digraphs for Example 5.12

Results in the remainder of this section focus on digraph aspects of the inverses of nonsingular nearly reducible matrices. For any digraph $D$, let $\bar{D}$ denote the transitive closure of $D$, i.e., the digraph obtained by adding to $D$ an $\operatorname{arc}(i, j)$ whenever $D$ contains a path from $i$ to $j(i \neq j)$ that is not the path $i \rightarrow j$ itself. For any digraph $D$ with no cycles of length greater than or equal to two, let $D^{\circ}$ denote the "transitive opening" of $D$, defined to be the digraph obtained by deleting from $D$ an arc $(i, j)$ whenever $D$ contains a path from $i$ to $j(i \neq j)$ that is not the path $i \rightarrow j$ itself. Note that if $D$ has no cycles of length greater than or equal to two, then $\bar{D}=\overline{D^{\circ}}$ and $D^{\circ}=(\bar{D})^{\circ}$.

LEMMA 5.13. If $A$ is a nonsingular nearly reducible matrix, and $P$ is a permutation matrix such that the matrix $A^{\prime}=P^{-1} A$ has only nonzero entries along the main diagonal, then $D\left(\left(A^{\prime}\right)^{-1}\right)=\overline{D\left(A^{\prime}\right)}$ and $D\left(A^{\prime}\right)=D\left(\left(A^{\prime}\right)^{-1}\right)^{\circ}$.

Proof. By Lemma 2.2 and Theorem 5.8, $D\left(\left(A^{\prime}\right)^{-1}\right)=\overline{D\left(A^{\prime}\right)}$. By Corollary 5.7, 
$D\left(A^{\prime}\right)$ is arc unique. Hence, $D\left(A^{\prime}\right)=D\left(A^{\prime}\right)^{\circ}=\left(\overline{D\left(A^{\prime}\right)}\right)^{\circ}=D\left(\left(A^{\prime}\right)^{-1}\right)^{\circ}$.

Let $D \in \mathcal{D}_{1}$ be a digraph with "next" and "former" permutations $n$ and $f$, respectively. For any digraph $D^{\prime}$ with vertex set $V\left(D^{\prime}\right)=V(D)$, let $N_{D} D^{\prime}$ and $D^{\prime} F_{D}$ denote the digraphs with vertex set $V(D)$ and respective arc sets

$$
\begin{aligned}
& E\left(N_{D} D^{\prime}\right)=\left\{(n(i), j) \mid(i, j) \in E\left(D^{\prime}\right)\right\} \quad \text { and } \\
& E\left(D^{\prime} F_{D}\right)=\left\{(i, f(j)) \mid(i, j) \in E\left(D^{\prime}\right)\right\} .
\end{aligned}
$$

The following lemma describes how these digraphs are related to digraphs of row (or column) permutations of an associated matrix.

Lemma 5.14. Let $M=\left[m_{i j}\right]$ be an $m \times m$ matrix and let $P=\left[p_{i j}\right]$ be an $m \times m$ permutation matrix. Then

$$
D(P M)=N_{D\left(P^{-1}\right)} D(M) \quad \text { and } \quad D(M P)=D(M) F_{D\left(P^{-1}\right)} .
$$

Proof. Let $n$ and $f$ be the "next" and "former" permutations in $D\left(P^{-1}\right)$. In other words, $n$ (resp. $f)$ is the permutation such that $p_{n(j) j}=1\left(\right.$ resp. $\left.p_{i f(i)}=1\right)$ for all $i$. Entry $(P M)_{i j}$ is nonzero if and only if both $p_{i k}$ and $m_{k j}$ are both nonzero, i.e., if the entry $m_{f(i) j}$ is nonzero. Thus for all $i, j,(i, j) \in E(D(P M))$ if and only if $(f(i), j) \in E(D(M))$. The latter is true if and only if $(i, j)=(n(f(i)), j)$ is an arc of $N_{D\left(P^{-1}\right)} D(M)$. Hence, $D(P M)=N_{D\left(P^{-1}\right)} D(M)$. The proof of the second identity is similar. $\square$

THEOREM 5.15. If $A$ is a nonsingular nearly reducible matrix, then the digraphs $D(A)$ and $D\left(A^{-1}\right)$ determine each other uniquely, as follows:

$$
D\left(A^{-1}\right)=\overline{N_{D(A)} D(A)} F_{D(A)} \quad \text { and } \quad D(A)=N_{D\left(A^{-1}\right)}\left(D\left(A^{-1}\right) F_{D\left(A^{-1}\right)}\right)^{\circ} .
$$

Proof. Let $P$ be a permutation matrix such that $P^{-1} A$ has only nonzero entries along its main diagonal. The nonzero diagonal in $A$ has the same coordinate positions as the nonzero diagonal in $P$, i.e., $\mathcal{C}_{D(A)}=\mathcal{C}_{D(P)}$. Thus by Lemmas 5.13 and 5.14,

$$
\begin{aligned}
D\left(A^{-1}\right) & =D\left(\left(P^{-1} A\right)^{-1} P^{-1}\right) \\
& =D\left(\left(P^{-1} A\right)^{-1}\right) F_{D(P)} \\
& =D\left(\left(P^{-1} A\right)^{-1}\right) F_{D(A)} \\
& =\overline{D\left(P^{-1} A\right)} F_{D(A)} \\
& =\overline{N_{D(P)} D(A)} F_{D(A)} \\
& =\overline{N_{D(A)} D(A)} F_{D(A)} .
\end{aligned}
$$

The second identity follows similarly.

Let $A$ be a nonsingular nearly reducible real matrix. By Corollary $5.9, A$ is strongly sign-nonsingular, so the sign pattern of $A$ determines the sign pattern of $A^{-1}$. The following result shows that the converse is also true, even though $A^{-1}$ need not be strongly sign-nonsingular. 


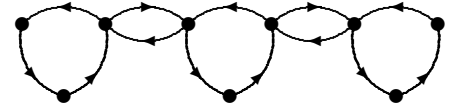

(a) $D$

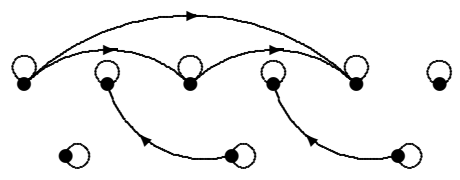

(c) $\overline{N_{D} D}$

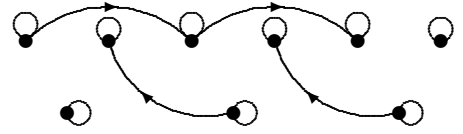

(b) $N_{D} D$

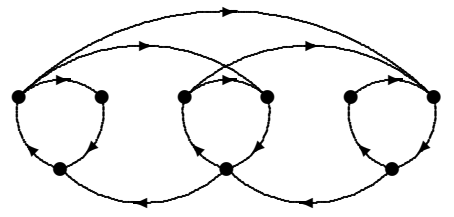

(d) $\overline{N_{D} D} F_{D}$

FIG. 5.2. Digraphs for Example 5.17

Proposition 5.16. If $B=\left[b_{i j}\right]$ is a nonsingular real matrix with a nearly reducible inverse, then without calculating $B^{-1}$ it is possible to determine the sign pattern of $B^{-1}$ directly from the sign pattern of $B$.

Proof. Let $A=\left[a_{i j}\right]=B^{-1}$. By Theorem 5.15, $D(A)=N_{D(B)}\left(D(B) F_{D(B)}\right)^{\circ}$, so the digraph $D(A)$ is determined by the digraph $D(B)$, which in turn is determined by the sign pattern of $B$. Thus, let $a_{r t}$ be a nonzero entry of $A$, and let $n$ and $f$ be the "next" and "former" permutations in $D(B)$. By Corollary 5.10, if $(t, r) \in E\left(\mathcal{C}_{D(B)}\right)$, then $a_{r t}=\frac{1}{b_{t r}}$; otherwise, $b_{f(r) n(t)}=\frac{-a_{r t}}{a_{r f(r)} a_{n(t) t}}$, giving $a_{r t}=-b_{f(r) n(t)} a_{r f(r)} a_{n(t) t}=$ $\frac{-b_{f(r) n(t)}}{b_{f(r) r} b_{t n(t)}}$. In either case, the sign of $a_{r t}$ is determined by the signs of entries of $B$. $\square$

EXAMPLE 5.17. If $A$ is a nearly reducible matrix, then without calculating $A^{-1}$ it is possible to determine the digraph $D\left(A^{-1}\right)$ directly from the digraph $D(A)$. The identity $D\left(A^{-1}\right)=\overline{N_{D(A)} D(A)} F_{D(A)}$ in Theorem 5.15 states explicitly how this may be done. To illustrate this procedure, Figure 5.2(a) displays the minimally strongly connected digraph $D$ from Figure 2.1. Suppose that $A$ is a (nearly reducible) matrix with $D(A)=D$. To determine $D\left(A^{-1}\right)$, first form the digraph $N_{D} D$ (see Figure 5.2(b)). Next, determine the transitive closure $\overline{N_{D} D}$ (see Figure 5.2(c)). Finally, determine the digraph $\overline{N_{D} D} F_{D}$, which equals $D\left(A^{-1}\right)$ (see Figure 5.2(d)). If $B$ is a matrix with $D(B)$ as in Figure 5.2(d) and if $B^{-1}$ is nearly reducible, then by Proposition 5.16, $D\left(B^{-1}\right)$ is as in Figure 5.2(a), and the sign pattern of $B^{-1}$ is determined by that of $B$.

If $A$ is a nonsingular nearly reducible matrix, then it follows from Theorem 5.15 that $A^{-1}$ has at least as many nonzero entries as $A$. Example 5.17 shows that $A^{-1}$ 
can in fact have more nonzero entries than $A$. A complete characterization of when the number of nonzero entries of $A$ and $A^{-1}$ are equal is given in Theorem 5.18 with a special case elaborated in Proposition 5.19.

THEOREM 5.18. If $A$ is a nonsingular nearly reducible matrix, then the following statements are equivalent:

1. $A^{-1}$ is nearly reducible;

2. $A^{-1}$ is strongly sign-nonsingular;

3. $A$ and $A^{-1}$ contain the same number of nonzero entries;

4. no cyclic sequence of $D(A)$ has length three;

5. no path in $N_{D(A)} D(A)$ has length two;

6. $N_{D(A)} D(A)=\overline{N_{D(A)} D(A)}$.

Proof. The equivalence of statements 4 and 5 follows from Lemma 2.2 since $N_{D(A)} D(A)=D\left(A^{\prime}\right)$, where $A^{\prime}$ is the matrix obtained by permuting the rows of $A$ such that the main diagonal of $A^{\prime}$ has only nonzero entries.

Statement 5 trivially implies statement 6 . By Corollary $5.7, D\left(A^{\prime}\right)$ is arc unique so statement 6 implies statement 5 .

Since $D(A)$ has the same number of arcs as $N_{D(A)} D(A) F_{D(A)}$, which is a subdigraph of $\overline{N_{D(A)} D(A)} F_{D(A)}=D\left(A^{-1}\right)$ by Theorem 5.15, $N_{D(A)} D(A)=\overline{N_{D(A)} D(A)}$ if and only if $D(A)$ and $D\left(A^{-1}\right)$ have the same number of arcs. Hence, statements 3 and 6 are equivalent.

To prove that statement 1 implies statement 4 , suppose that $D(A)$ contains a cyclic sequence $\left(v_{1}, \ldots, v_{s}\right)$ of length $s \geq 3$. Then $\left(v_{1}, v_{2}\right),\left(v_{2}, v_{3}\right)$, and $\left(v_{1}, v_{2}, v_{3}\right)$ are also cyclic sequences in $D(A)$, from $v_{1}$ to $f\left(v_{2}\right)$, from $v_{2}$ to $f\left(v_{3}\right)$, and from $v_{1}$ to $f\left(v_{3}\right)$, respectively. Thus by Theorem $5.8,\left(v_{1}, f\left(v_{2}\right)\right),\left(v_{2}, f\left(v_{3}\right)\right)$, and $\left(v_{1}, f\left(v_{3}\right)\right)$ are $\operatorname{arcs}$ of $D\left(A^{-1}\right)$. Hence,

$$
v_{1} \rightarrow f\left(v_{2}\right) \rightarrow f\left(f\left(v_{2}\right)\right) \rightarrow \cdots \rightarrow v_{2} \rightarrow f\left(v_{3}\right)
$$

is a path in $D\left(A^{-1}\right)$ from $v_{1}$ to $f\left(v_{3}\right)$. Since $\left(v_{1}, f\left(v_{3}\right)\right) \in E\left(D\left(A^{-1}\right)\right), D\left(A^{-1}\right)$ is not arc unique, so $A^{-1}$ is not nearly reducible by Corollary 5.7. Thus by taking the contrapositive, statement 1 implies statement 4 . To prove that statement 4 implies statement 1 , note that by the remarks after Corollary $5.10, D\left(A^{-1}\right)$ is strongly connected. Assume that $A^{-1}$ is not nearly reducible. Then by Lemma 5.1, $D\left(A^{-1}\right)$ is not arc unique, so there exists an arc $(u, v) \in E\left(D\left(A^{-1}\right)\right)$ and a path $u_{1} \rightarrow u_{2} \rightarrow$ $\cdots \rightarrow u_{t}$ with $u=u_{1}, v=u_{t}$ and $t \geq 3$. By Theorem 5.8 since $\left(u_{1}, u_{2}\right),\left(u_{2}, u_{3}\right) \in$ $E\left(D\left(A^{-1}\right)\right)$, there exists a cyclic sequence $\left(u_{1}, \ldots, n\left(u_{2}\right)\right)$ in $D(A)$ from $u_{1}$ to $u_{2}$, and a cyclic sequence $\left(u_{2}, \ldots, n\left(u_{3}\right)\right)$ in $D(A)$ from $u_{2}$ to $u_{3}$. Thus by Lemma $2.2, D\left(A^{\prime}\right)$ contains a path

$$
u_{1} \rightarrow \cdots \rightarrow u_{2} \rightarrow \cdots \rightarrow u_{3}
$$

Hence, $D\left(A^{\prime}\right)$ contains a path of length three, so by Lemma $2.2, D(A)$ contains a cyclic sequence of length three. Thus by taking the contrapositive, statement 4 implies statement 1 .

By Corollary 5.9, statement 1 immediately implies statement 2. To prove that statement 2 implies statement 1 , suppose that $A^{-1}$ is strongly sign-nonsingular. Since 
strong sign-nonsingularity is invariant with respect to row permutations, it may be assumed without loss of generality that the main diagonals of $A$ and $A^{-1}$ have only nonzero entries. By Theorem 5.15, $D\left(A^{-1}\right)=\overline{N_{D(A)} D(A)} F_{D(A)}=\overline{N_{D(A)} D(A)}$. Since by Theorem 4.3, $D\left(A^{-1}\right)$ is unipathic and thus arc unique, $N_{D(A)} D(A)=$ $\overline{N_{D(A)} D(A)}$. Hence statement 6 is true, which by the above implications implies that statement 1 is also true.

To illustrate the above theorem, consider the nearly reducible matrix $A$ in Figure 5.1. By Theorem 5.18, $A^{-1}$ is nearly reducible since $A$ and $A^{-1}$ each contain seven nonzero entries. In contrast, consider any nearly reducible matrix $A$ with digraph $D(A)=D$ as in Figure 5.2. By Theorem 5.18, $A^{-1}$ is neither strongly sign-nonsingular nor nearly reducible since $N_{D} D \neq \overline{N_{D} D}$.

Proposition 5.19. If $A$ is a nonsingular nearly reducible matrix, then

$$
D\left(A^{-1}\right)=D\left(A^{T}\right) \quad \text { if and only if } D(A) \text { is a cycle. }
$$

Proof. If $D(A)$ is a cycle, then clearly $D\left(A^{-1}\right)=D\left(A^{T}\right)$. To show that the converse implication is true, suppose that $D\left(A^{-1}\right)=D\left(A^{T}\right)$. Then $A$ and $A^{-1}$ have the same number of nonzero entries, so by Theorem 5.18 , the cyclic sequences in $D(A)$ have length either one or two. Assume that $(i, j) \in E(D(A))-E\left(\mathcal{C}_{D(A)}\right)$. Then $(j, i) \in E\left(D\left(A^{T}\right)\right)=E\left(D\left(A^{-1}\right)\right)$, so by Theorem 5.8 , there is a cyclic sequence from $j$ to $i$. Since $(i, j) \notin E\left(\mathcal{C}_{D(A)}\right)$, the cyclic sequence from $j$ to $i$ cannot have length one; thus it has length two. Hence, there exists an arc $(r, t) \in E(D(A))$ such that $(j, i)=(n(r), f(t))$, where $n$ and $f$ are the "next" and "former" permutations defined by $\mathcal{C}_{D(A)}$. Then

$$
r \rightarrow t \rightarrow n(t) \rightarrow \cdots \rightarrow f(t)=i \rightarrow j \rightarrow n(j) \rightarrow \cdots \rightarrow f(j)=r
$$

is a cycle in $D(A)$ that has $(r, j)$ as chord. This contradicts Lemma 5.3, so all arcs of $D(A)$ are contained in $E\left(\mathcal{C}_{D(A)}\right)$. In other words, $D(A)$ is a vertex-disjoint union of cycles. Since $D(A)$ is strongly connected, it must consist of a single cycle.

\section{REFERENCES}

[1] T. Britz, D. D. Olesky, and P. van den Driessche. The Moore-Penrose inverse of matrices with an acyclic bipartite graph. Linear Algebra Appl., to appear.

[2] R. A. Brualdi and M. B. Hedrick. A unified treatment of nearly reducible and nearly decomposable matrices. Linear Algebra Appl., 24:51-73, 1979.

[3] R. A. Brualdi and H. Ryser. Combinatorial Matrix Theory. Cambridge University Press, Cambridge, 1991

[4] R. A. Brualdi and B. L. Shader. Matrices of Sign-Solvable Linear Systems. Cambridge University Press, Cambridge, 1995.

[5] D. J. Hartfiel. A simplified form for nearly reducible and nearly decomposable matrices. Proc. Amer. Math. Soc., 24:388-393, 1970.

[6] F. Harary. A graph theoretic approach to matrix inversion by partitioning. Numer. Math., 4:128-135, 1962

[7] M. Hedrick and R. Sinkhorn. A special class of irreducible matrices - The nearly reducible matrices. J. Algebra, 16:143-150, 1970.

[8] D. E. Knuth. Wheels within wheels. J. Combin. Theory, Ser. B, 16:42-46, 1974. 
[9] T. Krahn. Theorems on arc unique digraphs. Report of a Summer Work Term at Department of Mathematics and Statistics and the Department of Computer Science at the University of Victoria, 1998

[10] R. D. Luce. Two decomposition theorems for a class of finite oriented graphs. Amer. J. Math., 74:701-722, 1952.

[11] J. S. Maybee. Some possible new directions for combinatorial matrix analysis. Linear Algebra Appl., 107:23-40, 1988.

[12] J. S. Maybee, D. D. Olesky, P. van den Driessche, and G. Wiener. Matrices, digraphs, and determinants. SIAM J. Matrix Anal. Appl., 10:500-519, 1989.

[13] C. Thomassen. When the sign pattern of a square matrix determines uniquely the sign pattern of its inverse. Linear Algebra Appl., 119:27-34, 1989. 